\title{
Identification of potential therapeutic targets for lung cancer by bioinformatics analysis
}

\author{
LI-QUAN WANG, LAN-HUA ZHAO and YI-ZE QIAO
}

\begin{abstract}
Department of Thoracic Surgery, Liaocheng People's Hospital and Liaocheng Clinical School of Taishan Medical University, Liaocheng, Shandong 252000, P.R. China
\end{abstract}

Received March 9, 2015; Accepted December 8, 2015

DOI: $10.3892 / \mathrm{mmr} .2015 .4752$

\begin{abstract}
The aim of the present study was to identify potential therapeutic targets for lung cancer and explore underlying molecular mechanisms of its development and progression. The gene expression profile datasets no. GSE3268 and GSE19804, which included five and 60 pairs of tumor and normal lung tissue specimens, respectively, were downloaded from Gene Expression Omnibus. Differentially expressed genes (DEGs) between lung cancer and normal tissues were identified, and gene ontology and Kyoto Encyclopedia of Genes and Genomes pathway analysis of the DEGs was performed. Furthermore, protein-protein interaction (PPI) networks and a transcription factor (TF) regulatory network were constructed and key target genes were screened. A total of 466 DEGs were identified, and the PPI network indicated that $I L-6$ and MMP 9 had key roles in lung cancer. A PPI module containing 34 nodes and 547 edges was obtained, including PTTG1. The TF regulatory network indicated that TFs of FOSB and LMO2 had a key role. Furthermore, $M M P 9$ was indicated to be the target of FOSB, while PTTG1 was the target of LMO2. In conclusion, the bioinformatics analysis of the present study indicated that IL-6, MMP9 and PTTG1 may have key roles in the progression and development of lung cancer and may potentially be used as biomarkers or specific therapeutic targets for lung cancer.
\end{abstract}

\section{Introduction}

Lung cancer is one of the most common malignancies and has a significant socioeconomic impact on patients and their families (1). In western countries, the mortality rate of lung cancer is $15 \%$ and the worldwide mortality rate for patients with lung cancer is $86 \%$ (2). The high mortality of lung cancer is mainly attributable to the lack of effective therapeutic methods and

Correspondence to: Dr Li-Quan Wang, Department of Thoracic Surgery, Liaocheng People's Hospital and Liaocheng Clinical School of Taishan Medical University, 67 Dongchang West Road, Liaocheng, Shandong 252000, P.R. China

E-mail: liquanwanglqw@163.com

Key words: lung cancer, protein-protein interaction network, differentially expressed gene the difficulty of obtaining an early diagnosis. Thus, the development of effective therapeutic targets is urgently required.

Differentially expressed genes (DEGs) have been reported to have important roles in lung cancer, and their identification may aid in the elucidation of its underlying molecular mechanisms as well as the discovery of novel biomarkers and treatments (3). Numerous genes, including p53 $(3,4)$, EGFR (5,6), kRAS (7), PIK3CA (8) and EML4 (9), are known to be associated with lung cancer, while others have remained elusive. Futhermore, SEMA5A and -6A were identified as potential therapeutic targets for lung cancer (10-12). Although tremendous efforts have been made to discover novel targets for lung cancer treatments, the current knowledge is insufficient and requires expansion.

In the present study, DEGs between lung cancer and normal lung tissues were identified. Protein-protein interaction (PPI) and transcription factor (TF) regulatory networks were constructed and key target genes were screened. Through the identification of key genes, the possible underlying molecular mechanisms as well as potential candidate biomarkers and treatment targets for lung cancer were explored.

\section{Materials and methods}

Affymetrix microarray data. The gene expression profile dataset no. GSE3268 deposited in the Gene Expression Omnibus (GEO) database (http://www.ncbi.nlm.nih.gov/geo/) by Wachi et al (13) based on the GPL96 platform (HG-U133A; Affymetrix Human Genome U133A Array), was subjected to bioinformatics analysis in the present study. The dataset contained a total of 10 chips, including five squamous cell lung cancer tissues and five paired adjacent normal lung tissues obtained from patients with squamous cell lung cancer.

Furthermore, the gene expression profile dataset GSE19804 based on the platform GPL570 (HG-U133_Plus_2; Affymetrix Human Genome U133 Plus 2.0 Array), which was deposited in the GEO database by Lu et al (14), was used. The dataset contained 120 chips, including 60 samples of non-small cell lung cancer tissues and 60 samples of paired normal lung tissues from female Taiwanese patients.

Identification of DEGs. The raw data were pre-processed using the Affy package (15) in R language. DEGs of GSE3268 (DEG1) and GSE19804 (DEG2) between normal groups and 
disease groups were respectively analyzed using the limma package in $\mathrm{R}$ (16). Fold changes (FCs) in the expression of individual genes were calculated and DEGs with $\mathrm{P}<0.05$ and $\mid \log \mathrm{FCl}>1$ were considered to be significant. DEG1 and DEG2 were then combined and the pooled dataset was referred to as the overlapping DEGs in the present study.

Gene ontology (GO) and pathway enrichment analysis of DEGs. GO analysis is a commonly used approach for functional studies of large-scale transcriptomic data (17). The Kyoto Encyclopedia of Genes and Genomes (KEGG) pathway database (18) contains information on networks of molecules or genes. The Database for Annotation, Visualization and Integrated Discovery (DAVID) (19) was used to systematically extract biological information from the large number of genes. GO functions and KEGG pathways of the overlapping DEGs were analyzed using DAVID 6.7 with $\mathrm{P}<0.05$.

Construction of PPI network and screening of modules. The Search Tool for the Retrieval of Interacting Genes (STRING) (20) database was used to retrieve the predicted interactions for the DEGs; version 9.1 of STRING covers 1,133 completely sequenced species. All associations obtained in STRING are provided with a confidence score, which represents a rough estimate of the likelihood of a given association to describe a functional linkage between two proteins (21). The overlapping DEGs with a confidence score $>0.4$ were selected to construct the PPI network using Cytoscape software (version 3.0; http://cytoscape.org/) (22). Cytoscape allows for the visualization of complex networks and their integration to any type of attribute data. The MCODE (23) plugin in Cytoscape was used to divide the PPI into modules. GO functional analysis of genes in the modules was performed using the BinGo 2.44 plugin in Cytoscape (24) with a threshold of $\mathrm{P}<0.05$ using the hypergeometric test.

Transcriptional regulatory network construction. The University of California at Santa Cruz (UCSC) database (http://genome.ucsc.edu) contains information on TF binding sites and the regulated genes (25). Using information collected from the UCSC database, DEGs were matched with their associated TFs. The TF regulatory network then was constructed using Cytoscape software (26).

\section{Results}

GO and pathway enrichment analysis of DEGs. From the GEO datasets, information on the expression of 8,172 genes was obtained. The normalized results showed that the expression median after normalization was in a straight line (Fig. 1). A total of 466 DEGs, including 156 upregulated and 310 downregulated genes, were selected.

Results of GO analysis showed that the upregulated DEGs were significantly enriched in biological processes, including collagen metabolic processes, multicellular organismal macromolecule metabolic processes and nuclear division (Table I); the downregulated DEGs were significantly enriched in biological processes, including response to wounding, immune response, defense response and inflammatory response (Table I).
Pathway analysis showed that the upregulated DEGs were significantly enriched in cell cycle, extracellular matrix - receptor interaction and the p53 signaling pathway (Table I); the downregulated DEGs were significantly enriched in cytokine receptor interaction, complement and coagulation cascades as well as chemokine signaling pathways (Table I).

Construction of PPI network and screening of module. The PPI network was constructed based on the predicted interactions of the identified DEGs (Fig. 2). Genes of IL-6, FOSB, $C D K 1, M M P 9$ and $I C A M 1$ were found to have a high degree of interaction in lung cancer. A sub-network containing 34 nodes and 547 edges was screened from the PPI network, such as PTTG1 (Fig. 3). The DEGs in the sub-net were significantly enriched in biological processes, such as the cell cycle, and pathway analysis showed that they were significantly enriched in cell cycle and oocyte meiosis (Table II).

TF-target gene regulatory network analysis. Associations between 44 TFs and their 47 target DEGs were collected from the TF regulatory network (Fig. 4). TFs of FOSB and LMO2, which exhibited a high degree of interaction, were selected from this network. Furthermore, the results also showed that $M M P 9$ was the target of FOSB and PTTG1 was the target of LMO2.

\section{Discussion}

Lung cancer is the leading cause of cancer-associated mortality; however, the underlying molecular mechanisms of its development and progression have remained to be fully elucidated (1). The present study used a bioinformatics approach to predict the potential therapeutic targets and explore the possible molecular mechanisms for lung cancer. A total of 466 DEGs between tumorous and normal tissues was identified, among which 310 genes were downregulated and 156 were upregulated. By constructing a PPI network and a TF regulatory network, key genes, including IL6, MMP9 and PTTG1, were identified.

IL-6 is a multifunctional cytokine that was characterized as a regulator of immune and inflammatory responses $(27,28)$. It is involved in the regulation of cell proliferation, survival and metabolism, and IL-6 signaling has an important role in tumorigenesis (29). Chung et al (30) found that IL-6 activated PI3K, which promoted apoptosis in human prostate cancer cell lines. Furthermore, studies have shown that IL-6 inhibited the growth of numerous types of cancer, including lung (31), breast (32) and prostate cancer (33). In the present study, $I L-6$ was shown to be downregulated in squamous cell and non-small cell lung cancer, and GO analysis showed that $I L-6$ was significantly enriched in biological processes, including defense response, inflammatory response, immune response and regulation of cell proliferation, which was consistent with a previous study (29). Combined with the above studies, it is indicated that $I L-6$ may be a diagnostic biomarker and therapeutic target in lung cancer.

MMP9 has a key role in cell migration, proliferation, differentiation, angiogenesis, apoptosis and host defense (34). Dysregulatoin of MMPs has been implicated in numerous diseases, including chronic ulcers and cancer (35-37). 

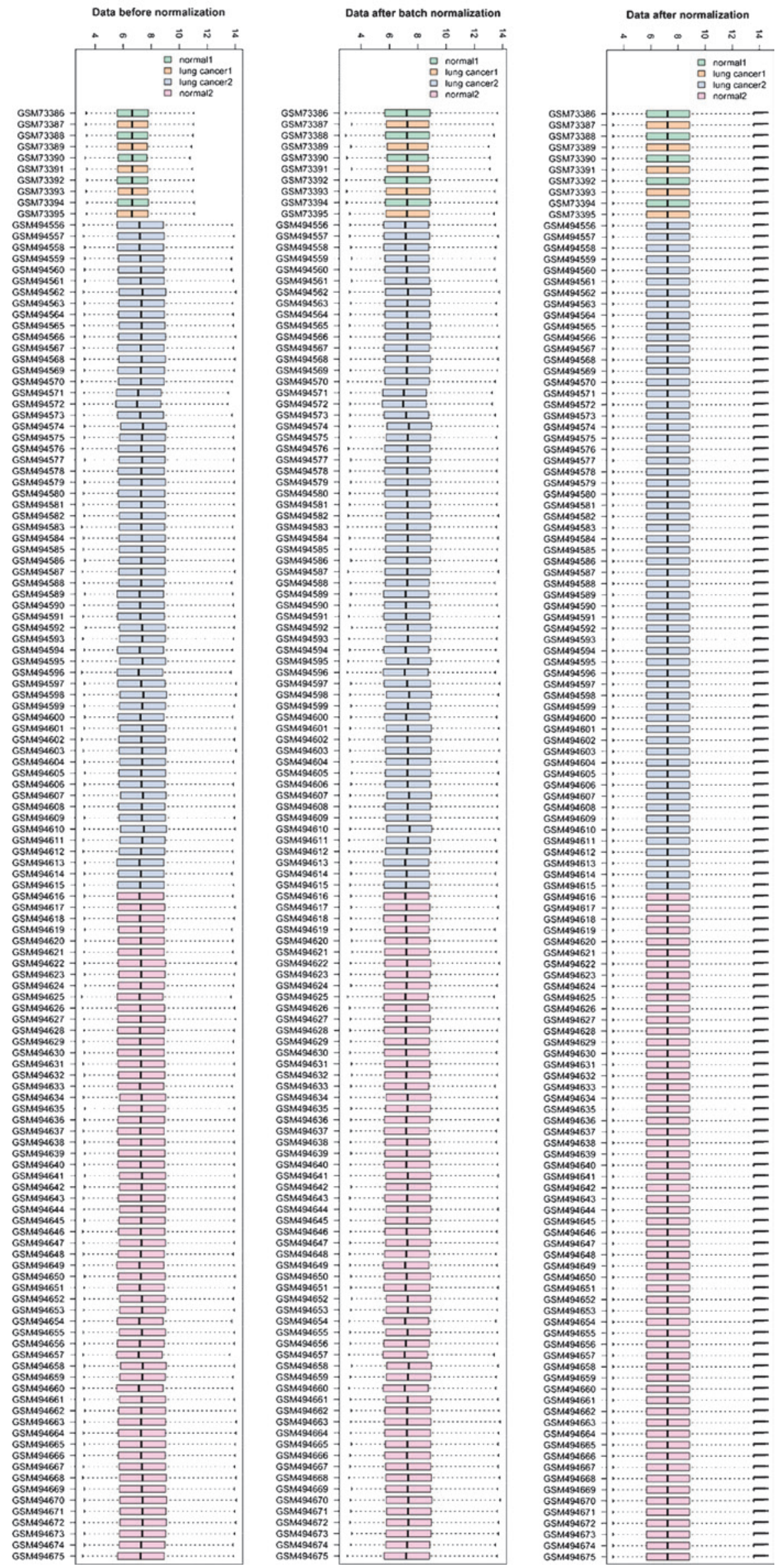

Figure 1. Boxplot of normalized expression values for the datasets. The dotted lines in the middle of each box represent the median of each sample, and its distribution among samples indicates the level of normalization of the data, with a nearly straight line indicating a fair normalization level. Gene expression omnibus datasets: 1, GSE3268; 2, GSE19804. 
Table I. GO and pathway analysis of the differentially expressed genes.

\begin{tabular}{|c|c|c|c|c|}
\hline Expression & Category & Term/gene and function & Count & P-value \\
\hline \multirow[t]{20}{*}{ Upregulated } & KEGG_PATHWAY & hsa04110 - Cell cycle & 12 & $6.94 \times 10^{-7}$ \\
\hline & KEGG_PATHWAY & hsa04512 - ECM-receptor interaction & 10 & $1.50 \times 10^{-6}$ \\
\hline & KEGG_PATHWAY & hsa04510 - Focal adhesion & 10 & $1.42 \times 10^{-3}$ \\
\hline & KEGG_PATHWAY & hsa04115 - p53 signaling pathway & 6 & $2.14 \times 10^{-3}$ \\
\hline & KEGG_PATHWAY & hsa00240 - Pyrimidine metabolism & 5 & $3.93 \times 10^{-2}$ \\
\hline & GOTERM_BP_FAT & GO:0032963 - Collagen metabolic process & 9 & $2.10 \times 10^{-10}$ \\
\hline & GOTERM_BP_FAT & $\begin{array}{l}\text { GO:0044259 - Multicellular organismal } \\
\text { macromolecule metabolic process }\end{array}$ & 9 & $5.19 \times 10^{-10}$ \\
\hline & GOTERM_BP_FAT & GO:0000280 - Nuclear division & 17 & $5.79 \times 10^{-10}$ \\
\hline & GOTERM_BP_FAT & GO:0007067 - Mitosis & 17 & $5.79 \times 10^{-10}$ \\
\hline & GOTERM_BP_FAT & GO:0000278 - Mitotic cell cycle & 21 & $7.04 \times 10^{-10}$ \\
\hline & GOTERM_BP_FAT & GO:0000087 - M phase of mitotic cell cycle & 17 & $7.55 \times 10^{-10}$ \\
\hline & GOTERM_CC_FAT & GO:0005576 - Extracellular region & 53 & $1.41 \times 10^{-10}$ \\
\hline & GOTERM_CC_FAT & GO:0005578 - Proteinaceous extracellular matrix & 19 & $7.80 \times 10^{-9}$ \\
\hline & GOTERM_CC_FAT & GO:0031012 - Extracellular matrix & 19 & $2.50 \times 10^{-8}$ \\
\hline & GOTERM_CC_FAT & GO:0044421 - Extracellular region part & 30 & $2.27 \times 10^{-7}$ \\
\hline & GOTERM_CC_FAT & GO:0005819 - Spindle & 12 & $4.55 \times 10^{-7}$ \\
\hline & GOTERM_MF_FAT & GO:0004222 - Metalloendopeptidase activity & 9 & $9.37 \times 10^{-6}$ \\
\hline & GOTERM_MF_FAT & GO:0048407 - Platelet-derived growth factor binding & 4 & $1.53 \times 10^{-4}$ \\
\hline & GOTERM_MF_FAT & GO:0004175 - Endopeptidase activity & 13 & $3.80 \times 10^{-4}$ \\
\hline & GOTERM_MF_FAT & GO:0004857 - Enzyme inhibitor activity & 11 & $3.81 \times 10^{-4}$ \\
\hline \multirow[t]{20}{*}{ Downregulated } & KEGG_PATHWAY & hsa04060 - Cytokine-cytokine receptor interaction & 20 & $6.99 \times 10^{-5}$ \\
\hline & KEGG_PATHWAY & hsa04610 - Complement and coagulation cascades & 8 & $2.47 \times 10^{-3}$ \\
\hline & KEGG_PATHWAY & hsa04062 - Chemokine signaling pathway & 13 & $4.53 \times 10^{-3}$ \\
\hline & KEGG_PATHWAY & hsa04650 - Natural killer cell mediated cytotoxicity & 10 & $9.69 \times 10^{-3}$ \\
\hline & KEGG_PATHWAY & hsa04614 - Renin-angiotensin system & 4 & $1.01 \times 10^{-2}$ \\
\hline & GOTERM_BP_FAT & GO:0009611 - Response to wounding & 48 & $2.23 \times 10^{-17}$ \\
\hline & GOTERM_BP_FAT & GO:0006952 - Defense response & 46 & $1.66 \times 10^{-13}$ \\
\hline & GOTERM_BP_FAT & GO:0006954 - Inflammatory response & 33 & $2.92 \times 10^{-13}$ \\
\hline & GOTERM_BP_FAT & GO:0006955 - Immune response & 43 & $4.20 \times 10^{-10}$ \\
\hline & GOTERM_BP_FAT & GO:0048545 - Response to steroid hormone stimulus & 21 & $3.81 \times 10^{-9}$ \\
\hline & GOTERM_CC_FAT & GO:0005615 - Extracellular space & 55 & $2.36 \times 10^{-18}$ \\
\hline & GOTERM_CC_FAT & GO:0044421 - Extracellular region part & 64 & $2.03 \times 10^{-17}$ \\
\hline & GOTERM_CC_FAT & GO:0005576 - Extracellular region & 93 & $3.37 \times 10^{-15}$ \\
\hline & GOTERM_CC_FAT & GO:0005886 - Plasma membrane & 131 & $2.25 \times 10^{-12}$ \\
\hline & GOTERM_CC_FAT & GO:0005887 - Integral to plasma membrane & 61 & $1.99 \times 10^{-11}$ \\
\hline & GOTERM_MF_FAT & GO:0019838 - Growth factor binding & 16 & $2.01 \times 10^{-9}$ \\
\hline & GOTERM_MF_FAT & GO:0030246 - Carbohydrate binding & 27 & $7.86 \times 10^{-9}$ \\
\hline & GOTERM_MF_FAT & GO:0019955 - Cytokine binding & 13 & $1.54 \times 10^{-6}$ \\
\hline & GOTERM_MF_FAT & GO:0005509 - Calcium ion binding & 39 & $1.04 \times 10^{-5}$ \\
\hline & GOTERM_MF_FAT & GO:0030247 - Polysaccharide binding & 14 & $1.11 \times 10^{-5}$ \\
\hline
\end{tabular}

BP, biological process; CC, cellular component; MF, molecular function; Count, numbers of differentially expressed genes; ECM, extracellular matrix; GO, gene ontology; hsa, Homo sapiens; KEGG, Kyoto Encyclopedia of Genes and Genomes; FAT, functional annotation tool.

Downregulation of MMPs has been shown to inhibit metastasis, while upregulation of MMPs led to enhanced cancer cell invasion (37). In the present study, MMP9 was overexpressed and regulated by $F O S B$ in lung cancer tissues. Kim et al (38) found that $F O S B$ was downregulated in pancreatic cancer and promoted tumor progression. Kataoka et al (39) found that FOSB gene expression in cancer stroma is a independent prognostic indicator for patients with epithelial ovarian cancer receiving standard therapy. Combined with the above studies, the present study indicated that MMP9 may have important roles in the progression of lung cancer, and that it may be utilized as a therapeutic target. 


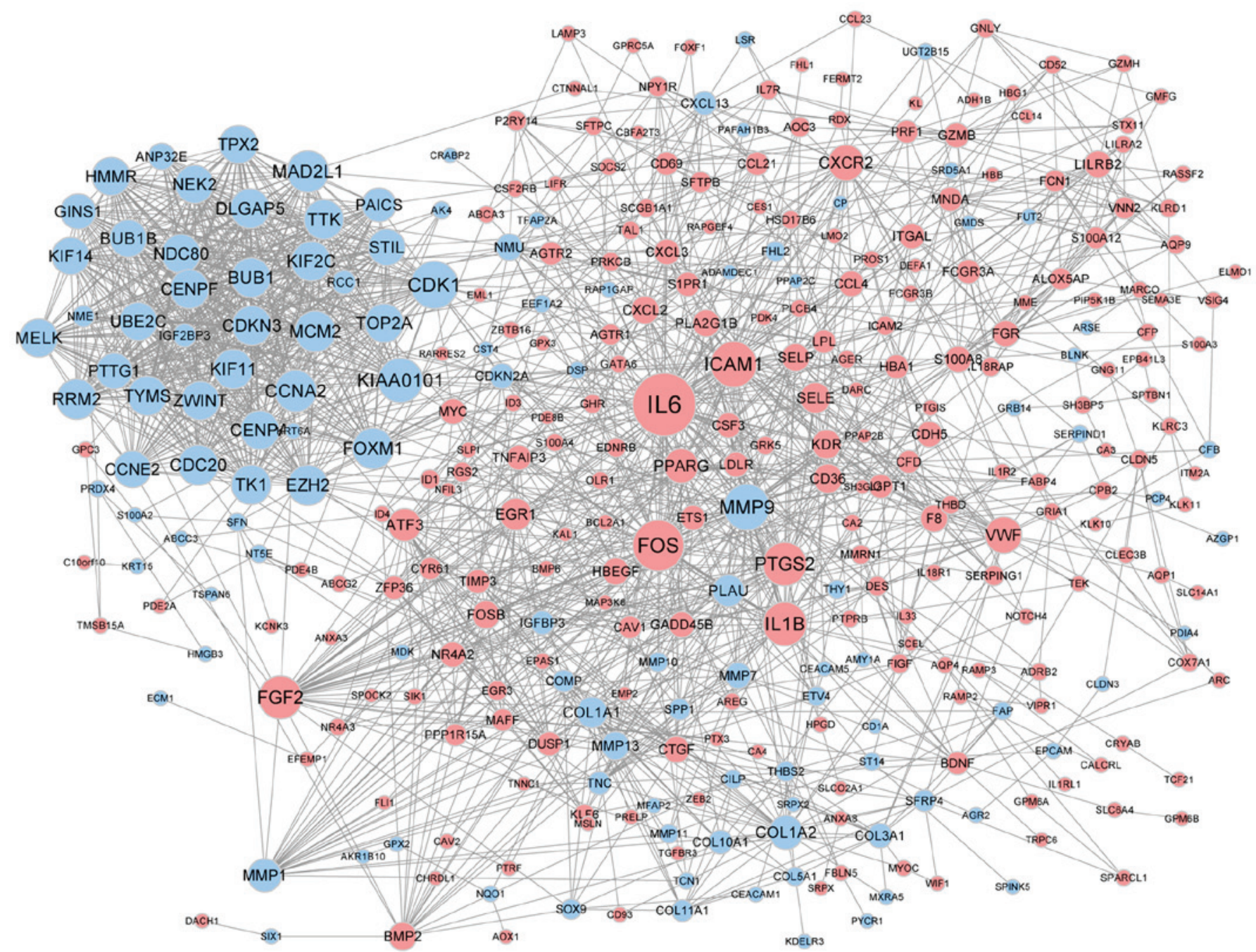

Figure 2. Protein-protein interaction network of the DEGs. Blue nodes represent products of upregulated DEGs and pink nodes represent products of downregulated DEGs. The size of each node is proportional to the degree of nodes. DEG, differentially expressed gene.

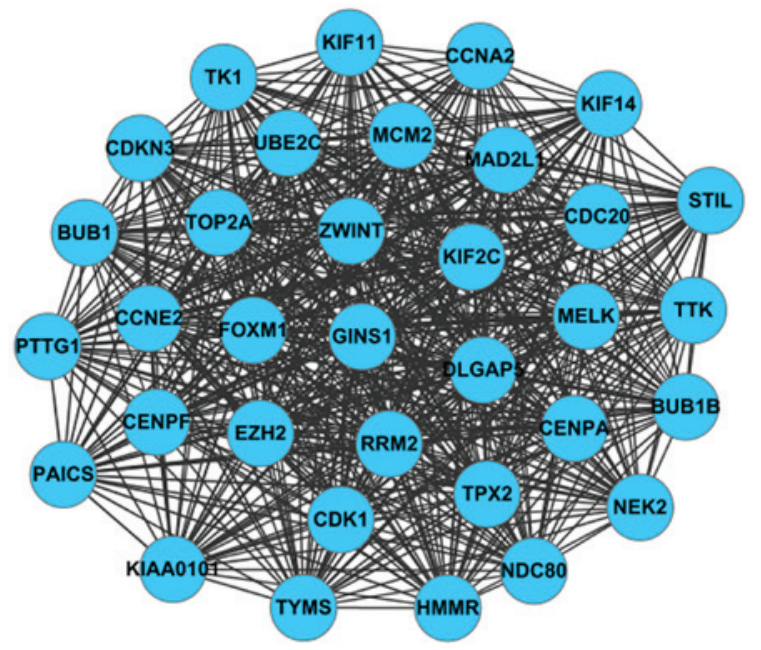

Figure 3. Sub-network screened from protein-protein interaction network. Nodes refer to the products of upregulated differentially expressed genes.

PTTG1 has tumorigenic activity and is highly expressed in various tumor types (40). Studies have shown that PTTG1 was overexpressed in esophageal cancer and associated with endocrine therapy resistance in breast cancer $(41,42)$. Yoon et al (40) showed that the PTTG1 oncogene promoted tumor malignancy via epithelial-to-mesenchymal expansion of the cancer stem cell population. Hamid et al (43) found that PTTG1 promoted tumorigenesis in human embryonic kidney cells. A study by Li et al (44) indicated that PTTG1 promoted migration and invasion of human non-small cell lung cancer cells. Panguluri et al (45) showed that PTTG1 was an important target gene for ovarian cancer therapy. In the present study, PTTG1 was found to be overexpressed in lung cancer tissues and regulated by LMO2. LMO2 is an important regulator in determining cell fate and controlling cell growth and differentiation (46). Nakata et al (47) found that $L M O 2$ was a novel predictive biomarker with the potential to enhance the accuracy of prognoses for pancreatic cancer. Yamada et al (48) showed that LMO2 is a key regulator of tumour angiogenesis. Combined with the above studies, the present study indicated that PTTG1 may have important roles in the progression of lung cancer and that it may represent a therapeutic target.

In conclusion, the bioinformatics analysis of the present study indicated that $I L-6, M M P 9$ and PTTG1 may have key roles in the progression and development of lung cancer. They 
Table II. GO and pathway analysis of genes in sub-network.

\begin{tabular}{|c|c|c|c|}
\hline Category & Term/gene and function & Count & P-value \\
\hline KEGG_PATHWAY & hsa04110 - Cell cycle & 10 & $1.09 \times 10^{-11}$ \\
\hline KEGG_PATHWAY & hsa04114- Oocyte meiosis & 6 & $1.09 \times 10^{-5}$ \\
\hline KEGG_PATHWAY & hsa04914 - Progesterone-mediated oocyte maturation & 4 & $1.83 \times 10^{-3}$ \\
\hline KEGG_PATHWAY & hsa04115 - p53 signaling pathway & 3 & $1.65 \times 10^{-3}$ \\
\hline KEGG_PATHWAY & hsa00240 - Pyrimidine metabolism & 3 & $3.10 \times 10^{-2}$ \\
\hline GOTERM_BP_FAT & GO:0000278 - Mitotic cell cycle & 19 & $7.13 \times 10^{-21}$ \\
\hline GOTERM_BP_FAT & GO:0007049 - Cell cycle & 22 & $1.65 \times 10^{-19}$ \\
\hline GOTERM_BP_FAT & GO:0000280 - Nuclear division & 16 & $2.14 \times 10^{-19}$ \\
\hline GOTERM_BP_FAT & GO:0007067 - Mitosis & 16 & $2.14 \times 10^{-19}$ \\
\hline GOTERM_BP_FAT & GO:0000087 - M phase of mitotic cell cycle & 16 & $2.82 \times 10^{-19}$ \\
\hline GOTERM_CC_FAT & GO:0005819 - Spindle & 12 & $9.20 \times 10^{-15}$ \\
\hline GOTERM_CC_FAT & GO:0000777 - Condensed chromosome kinetochore & 8 & $3.94 \times 10^{-11}$ \\
\hline GOTERM_CC_FAT & GO:0015630 - Microtubule cytoskeleton & 14 & $5.31 \times 10^{-11}$ \\
\hline GOTERM_CC_FAT & GO:0000779 - Condensed chromosome, centromeric region & 8 & $1.01 \times 10^{-10}$ \\
\hline GOTERM_CC_FAT & GO:0000922 - Spindle pole & 7 & $1.01 \times 10^{-10}$ \\
\hline GOTERM_MF_FAT & GO:0005524 - Adenosine triphosphate binding & 15 & $4.89 \times 10^{-7}$ \\
\hline GOTERM_MF_FAT & GO:0032559 - Adenyl ribonucleotide binding & 15 & $5.78 \times 10^{-7}$ \\
\hline GOTERM_MF_FAT & GO:0030554 - Adenyl nucleotide binding & 15 & $1.10 \times 10^{-6}$ \\
\hline GOTERM_MF_FAT & GO:0001883 - Purine nucleoside binding & 15 & $1.32 \times 10^{-6}$ \\
\hline GOTERM_MF_FAT & GO:0001882 - Nucleoside binding & 15 & $1.44 \times 10^{-6}$ \\
\hline
\end{tabular}

BP, biological process; CC, cellular component; MF, molecular function; Count, numbers of DEGs; GO, gene ontology; hsa, Homo sapiens; KEGG, Kyoto Encyclopedia of Genes and Genomes; FAT, functional annotation tool.

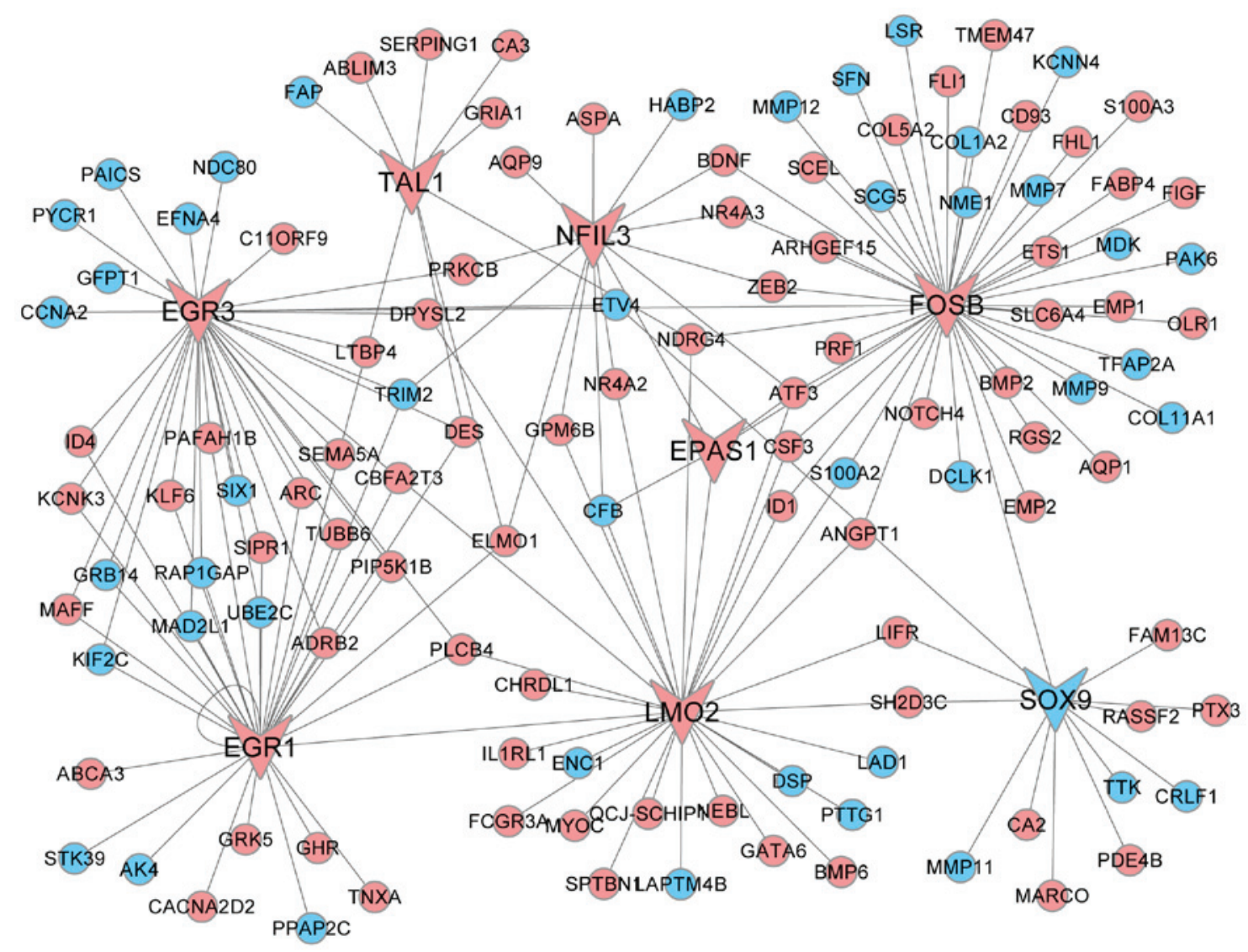

Figure 4. Transcriptional regulatory network analysis. Blue nodes represent products of upregulated DEGs and pink nodes represent products of downregulated DEGs. Triangle arrowheads indicate transcription factors and circles indicate target genes. DEG, differentially expressed gene. 
may be used as prognostic biomarkers as well as specific therapeutic targets for the treatment of lung cancer. However, molecular biology experiments are required to confirm these findings.

\section{References}

1. Nugent M, Edney B, Hammerness PG, Dain BJ, Maurer LH and Rigas JR: Non-small cell lung cancer at the extremes of age: Impact on diagnosis and treatment. Ann Thorac Surg 63: 193-197, 1997.

2. Yang SP,Luh KT, Kuo SH and Lin CC: Chronological observation of epidemiological characteristics of lung cancer in Taiwan with etiological consideration-a 30-year consecutive study. Jpn J Clin Oncol 14: 7-19, 1984.

3. Andriani F, Roz E, Caserini R, Conte D, Pastorino U, Sozzi G, and Roz L: Inactivation of both FHIT and p53 cooperate in deregulating proliferation-related pathways in lung cancer. J Thorac Oncol 7: 631-642, 2012.

4. Toyooka S, Tsuda T and Gazdar AF: The TP53 gene, tobacco exposure and lung cancer. Hum Mutat 21: 229-239, 2003.

5. Shigematsu H, Lin L, Takahashi T, Nomura M, Suzuki M, Wistuba II, Fong KM, Lee H, Toyooka S, Shimizu N, et al: Clinical and biological features associated with epidermal growth factor receptor gene mutations in lung cancers. J Nat Cancer Inst 97: 339-346, 2005.

6. Martin P, Kelly CM and Carney D: Epidermal growth factor receptor-targeted agents for lung cancer. Cancer Control 13 129-140, 2006.

7. Eberhard DA, Johnson BE, Amler LC, Goddard AD, Heldens SL, Herbst RS, Ince WL, Jänne PA, Januario T, Johnson DH, et al: Mutations in the epidermal growth factor receptor and in KRAS are predictive and prognostic indicators in patients with non-small-cell lung cancer treated with chemotherapy alone and in combination with erlotinib. J Clin Oncol 23: 5900-5909, 2005.

8. Yamamoto H, Shigematsu H, Nomura M, Lockwood WW Sato M, Okumura N, Soh J, Suzuki M, Wistuba II, Fong KM, et al: PIK3CA mutations and copy number gains in human lung cancers. Cancer Res 68: 6913-6921, 2008.

9. Wong DW, Leung EL, So KK, Tam IY, Sihoe AD, Cheng LC Ho KK, Au JS, Chung LP, Pik Wong M, et al: The EML4-ALK fusion gene is involved in various histologic types of lung cancers from nonsmokers with wild-type EGFR and KRAS. Cancer 115 1723-1733, 2009.

10. Castro-Rivera E, Ran S, Thorpe P and Minna JD: Semaphorin 3B (SEMA3B) induces apoptosis in lung and breast cancer, whereas VEGF165 antagonizes this effect. Proc Natl Acad Sci USA 101: 11432-11437, 2004.

11. Tomizawa Y, Sekido Y, Kondo M, Gao B, Yokota J, Roche J, Drabkin H, Lerman MI, Gazdar AF, Minna JD, et al: Inhibition of lung cancer cell growth and induction of apoptosis after reexpression of 3p21. 3 candidate tumor suppressor gene SEMA3B. Proc Natl Acad Sci USA 98: 13954-13959, 2001.

12. Brambilla E, Constantin B, Drabkin H and Roche J: Semaphorin SEMA3F localization in malignant human lung and cell lines: A suggested role in cell adhesion and cell migration. Am J Pathol 156: 939-950, 2000

13. Wachi S, Yoneda $\mathrm{K}$ and Wu R: Interactome-transcriptome analysis reveals the high centrality of genes differentially expressed in lung cancer tissues. Bioinformatics 21: 4205-4208, 2005

14. Lu TP, Tsai MH, Lee JM, Hsu CP, Chen PC, Lin CW, Shih JY, Yang PC, Hsiao CK, Lai LC, et al: Identification of a novel biomarker, SEMA5A, for non-small cell lung carcinoma in nonsmoking women. Cancer Epidemiol Biomarkers Prev 19: 2590-2597, 2010.

15. Gautier L, Cope L, Bolstad BM and Irizarry RA: Affy-analysis of Affymetrix GeneChip data at the probe level. Bioinformatics 20: 307-315, 2004

16. Smyth GK: Linear models and empirical bayes methods for assessing differential expression in microarray experiments. Stat Appl Genet Mol Biol 3: 2004.

17. Hulsegge I, Kommadath A and Smits MA: Globaltest and GOEAST: Two different approaches for Gene Ontology analysis. BMC Proc 3 (Suppl 4): S10, 2009.

18. Ogata H, Goto S, Sato K, Fujibuchi W, Bono H and Kanehisa M KEGG: Kyoto encyclopedia of genes and genomes. Nucleic Acids Res 27: 29-34, 1999.
19. Dennis G Jr, Sherman BT, Hosack DA, Yang J, Gao W, Lane HC and Lempicki RA: DAVID: Database for annotation, visualization and integrated discovery. Genome Biol 4: P3, 2003.

20. Franceschini A, Szklarczyk D, Frankild S, Kuhn M, Simonovic M, Roth A, Lin J, Minguez P, Bork P, von Mering C, et al: STRING v9.1: Protein-protein interaction networks, with increased coverage and integration. Nucleic Acids Res 41: D808-D815, 2013.

21. Szklarczyk D, Franceschini A, Kuhn M, Simonovic M, Roth A Minguez P, Doerks T, Stark M, Muller J, Bork P, et al: The STRING database in 2011: Functional interaction networks of proteins, globally integrated and scored. Nucleic Acids Res 39: D561-D568, 2011.

22. Kohl M, Wiese S and Warscheid B: Cytoscape: Software for visualization and analysis of biological networks. Methods Mol Biol 696: 291-303, 2011

23. Bader GD and Hogue CW: An automated method for finding molecular complexes in large protein interaction networks. BMC Bioinformatics 4: 2, 2003.

24. Maere S, Heymans K and Kuiper M: BiNGO: A cytoscape plugin to assess overrepresentation of gene ontology categories in biological networks. Bioinformatics 21: 3448-3449, 2005.

25. Wingender E, Dietze P, Karas H and Knüppel R: TRANSFAC: A database on transcription factors and their DNA binding sites. Nucleic Acids Res 24: 238-241, 1996.

26. Shannon P, Markiel A, Ozier O, Baliga NS, Wang JT, Ramage D, Amin N, Schwikowski B and Ideker T: Cytoscape: A software environment for integrated models of biomolecular interaction networks. Genome Res 13: 2498-2504, 2003.

27. Schafer ZT and Brugge JS: IL-6 involvement in epithelial cancers. J Clin Invest 117: 3660-3663, 2007.

28. Kishimoto T: Interleukin-6: From basic science to medicine-40 years in immunology. Annu. Rev Immunol 23: 1-21, 2005.

29. Hodge DR, Hurt EM and Farrar WL: The role of IL-6 and STAT3 in inflammation and cancer. Eur J Cancer 41: 2502-2512, 2005.

30. Chung TD, Yu JJ, Kong TA, Spiotto MT and Lin JM: Interleukin-6 activates phosphatidylinositol-3 kinase, which inhibits apoptosis in human prostate cancer cell lines. Prostate 42: 1-7, 2000.

31. Takizawa H, Ohtoshi T, Ohta K, Yamashita N, Hirohata S, Hirai K, Hiramatsu K and Ito K: Growth inhibition of human lung cancer cell lines by interleukin 6 in vitro: A possible role in tumor growth via an autocrine mechanism. Cancer Res 53: 4175-4181, 1993

32. Knüpfer H and Preiss R: Significance of interleukin-6 (IL-6) in breast cancer (review). Breast Cancer Res Treat 102: 129-135, 2007.

33. Giri D, Ozen M and Ittmann M: Interleukin-6 is an autocrine growth factor in human prostate cancer. Am J Pathol 159: 2159-2165, 2001

34. Sica A, Allavena P and Mantovani A: Cancer related inflammation: The macrophage connection. Cancer Lett 267: 204-215, 2008.

35. Benveniste EN: Role of macrophages/microglia in multiple sclerosis and experimental allergic encephalomyelitis. J Mol Med (Berl) 75: 165-173, 1997.

36. Firestein GS: Evolving concepts of rheumatoid arthritis. Nature 423: 356-361, 2003.

37. Coussens LM, Fingleton B and Matrisian LM: Matrix metalloproteinase inhibitors and cancer: Trials and tribulations. Science 295: 2387-2392, 2002.

38. Kim JH, Lee JY, Lee KT, Lee JK, Lee KH, Jang KT, Heo JS, Choi SH and Rhee JC: RGS16 and FosB underexpressed in pancreatic cancer with lymph node metastasis promote tumor progression. Tumor Biol 31: 541-548, 2010.

39. Kataoka F, Tsuda H, Arao T, Nishimura S, Tanaka H, Nomura H, Chiyoda T, Hirasawa A, Akahane T, Nishio H, et al: EGRI and FOSB gene expressions in cancer stroma are independent prognostic indicators for epithelial ovarian cancer receiving standard therapy. Gene Chromosome Cancer 51: 300-312, 2012.

40. Yoon CH, Kim MJ, Lee H, Kim RK, Lim EJ, Yoo KC, Lee GH, Cui YH, Oh YS, Gye MC, et al: PTTG1 oncogene promotes tumor malignancy via epithelial to mesenchymal transition and expansion of cancer stem cell population. J Biol Chem 287: 19516-19527, 2012.

41. Shibata Y, Haruki N, Kuwabara Y, Nishiwaki T, Kato J, Shinoda N, Sato A, Kimura M, Koyama H, Toyama T, et al: Expression of PTTG (pituitary tumor transforming gene) in esophageal cancer. Jpn J Clin Oncol 32: 233-237, 2002. 
42. Ghayad SE, Vendrell JA, Bieche I, Spyratos F, Dumontet C, Treilleux I, Lidereau R and Cohen PA: Identification of TACC1, NOV and PTTG1 as new candidate genes associated with endocrine therapy resistance in breast cancer. J Mol Endocrinol 42: 87-103, 2009.

43. Hamid T, Malik MT and Kakar SS: Ectopic expression of PTTG1/securin promotes tumorigenesis in human embryonic kidney cells. Mol Cancer 4: 3, 2005.

44. Li H, Yin C, Zhang B, Sun Y, Shi L, Liu N, Liang S, Lu S, Liu Y, Zhang J, et al: PTTG1 promotes migration and invasion of human non-small cell lung cancer cells and is modulated by miR-186. Carcinogenesis 34: 2145-2155, 2013.
45. Panguluri SK, Yeakel C and Kakar SS: PTTG: An important target gene for ovarian cancer therapy. J Ovarian Res 1: 6, 2008.

46. Ma S, Guan XY, Beh PS, Wong KY, Chan YP, Yuen HF, Vielkind $\mathrm{J}$ and Chan KW: The significance of LMO2 expression in the progression of prostate cancer. J Pathol 211: 278-285, 2007.

47. Nakata K, Ohuchida K, Nagai E, Hayashi A, Miyasaka Y, Kayashima T, Yu J, Aishima S, Oda Y, Mizumoto K, et al: LMO2 is a novel predictive marker for a better prognosis in pancreatic cancer. Neoplasia 11: 712-719, 2009.

48. Yamada Y,Pannell R,Forster A and Rabbitts TH: The LIM-domain protein Lmo2 is a key regulator of tumour angiogenesis: A new anti-angiogenesis drug target. Oncogene 21: 1309-1315, 2002. 